\title{
Challenges To Performance Testing Of The Cloud Application Developing
}

\author{
Yue Zhou \\ National Application Testing Labs \\ Beijing, China \\ e-mail: zhouyue@bsw.net.cn
}

\author{
Wenchuang Qin, Nafei Zhu \\ National Application Testing Labs \\ Beijing, China \\ e-mail: qinwenchuang@bsw.net.cn \\ zhunf@bsw.net.cn
}

\begin{abstract}
When we perform a Cloud Application performance testing, traditional performance testing tool and method is limited by many characteristics which are different between Cloud Computing and traditional application. This article descripts the limitation and weak point of traditional tool and method for performance testing on a Cloud Computing application. Face to challenge, some new methodologies are introduced.
\end{abstract}

Keywords-component; Cloud Computing; Performance Testing; Method

\section{INTRODUCTION}

Cloud computing is recognized to be another major information technology innovation after the personal computer and the Internet. China's national economic and social development plan make the cloud computing which is the representative of a new generation for information technology be key development for the strategic emerging industry. With cloud computing mode for service characteristics in all applications will get rapid development [1].

First, three operators of telecom industry will speed up the implementation of cloud computing strategy to achieve business innovation with the aid of cloud computing.

Second, in the financial industry small and medium-sized financial industry force set up public cloud service center.

Third, government promotes the reform of electronic government through the cloud computing platform.

Fourth, education industry improves resource utilization by establishing sharing cloud platform.

Along with the vigorous development of clouds application which is different from the traditional application, it is the need to find the new way for performance testing. Performance testing faces many challenges under the cloud computing service mode.

\section{Relative To The Vigorous DeVElopment Of The Cloud Computing Service, Traditional Performance TEST Mode Has Fallen BEHIND.}

Relative to the cloud computing service applications, server resources and network resources of the traditional application are tangibly and visible, and it is the concentrate deployment of data and resources. For the traditional application, the automation testing tool can efficiently do the performance testing instead of artificial nowadays, but in the face of cloud computing service applications which are highly transparent and large scale in system, the traditional performance testing tools and methods become backward.

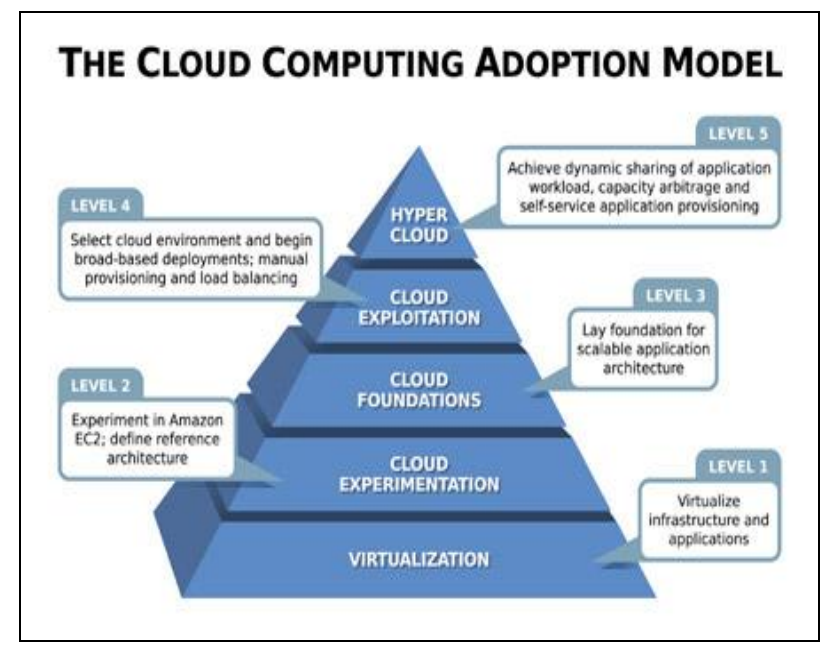

Figure 1. Schematic diagram for cloud computing service

The above is the schematic diagram for cloud computing service, with the network becoming more and more complicated and isomerization, especially with the vigorous development of virtualization technology, distributed computing, distributed storage and other new technology development under the background of cloud computing, the application mode of cloud computing service becomes more and more transparent and enormous. Most of the time, not only the measured object is huge in number, but also even the measured object of clouds application is intangible and invisible. The traditional testing method and tool face great challenges.

Dennis Drogseth, the vice President of market research company Enterprise Management Associates, says, for the application in the cloud environment, "you may need test the network performance, server performance, database performance, software performance, and their situations of the client cache. If an application runs only in one position, of course you test its performance only in this point. But, as to Amazon or for Facebook whose applications are distributed in many different and unpredictable positions, the work for performance testing is obviously much more complicated than the one of running the test scripts on a single server."

Traditional performance testing methods is limited for cloud computing services performance testing.

\section{A. Simulation of Massive Clouds Pressure Will Be High Price and Even Cannot Be Simulated.}

At present, the number of simulation users of performance test tools is restricted by the constraints of the license 
agreement. For example, LoadRunner, the fee for the license of tens of thousands levels often as high as hundreds dollar, and for the system of railway ticket, civil service examination registration and www.ebuy.com applications always need hundreds of thousands or even millions of user pressure, which make this kind of test tools incompetent. Even if the license allows, the number of pressure deployed is also a terrible. Assume there is a server with 4 core CPUs and 8G memory can generate 10000 virtual users online for simulation script pressure. But for the transparent and unified clouds application system usually need dozens or even hundreds of pressure platform, thus many sets of pressure press the clouds and make the testing cost very large.

\section{B. The Traditional Test Tool is Difficult To Get The Real} User Experience by Traversing The Complex Access Paths.

Performance testing results under traditional data centralized cases often reflect more about the performance of the system itself and it is not enough as to the user's real experience status. For the clouds service application mode, server, data and storage resources are transparent. Service providers concern not only the performance of the system itself, but also the user's experience which is the QoS index for service provider. As to the end users, obtaining good QoS experience is the source power to accept cloud service.

At present, performance testing tool can simulate the user access behavior, but the script record is often an access path at certain time, which can't represent the entire user's access path, so the script development and deployment tools will face huge cost if the current script will traverse the user's access paths.

C. Performance Testing Will Be More Dependent On The Cooperation Of Network Operators and Cdn[2] Service Business.

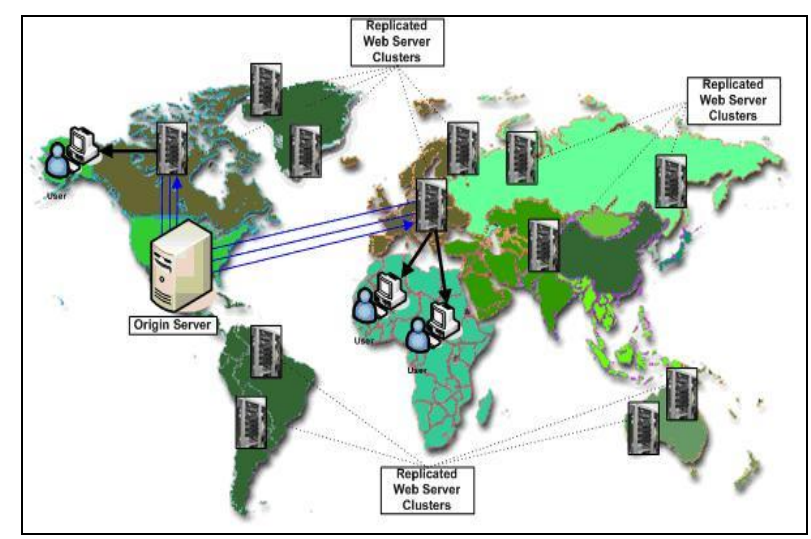

Figure 2. Abstract architecture of a Content Delivery Network (CDN)

Fig. 2 shows a typical content delivery environment where the replicated Web server clusters are located at the edge of the network to which the end-users are connected. In such CDN environment, Web content based on user requests are fetched from the origin server and a user is served with the content from the nearby replicated web server. Thus the user ends up communicating with a replicated CDN server close to it and retrieves files from that server. This kind of structure system is a typical representative. We can see that compared with the original data center mode with non-CDN technology, the current cloud service application of the whole ecology chain is integrated into more manufacturers and application, for which network operators and $\mathrm{CDN}$ service provider is an inevitable mechanism to large cloud computing server application. Performance testing also needs this cooperation of many parts. Such as the restriction and coordination for testing network bandwidth, the restrictions and coordination for IP analog, access security issues, and test isolation with other cloud service, etc.

\section{Cloud Service Performance Testing Methods FOR INDUSTRY SCENES NOWADAYS}

\section{A. Complete Simulation For The Clouds Pressure Testing}

Testing results using this method is the most useful as a reference. But for large the clouds application, such as www.taobao.com system, public ticketing system, and traditional performance testing tool Loadrunner can't simulate the mass users. Currently, the license of Loadrunner is limitation, which is unable to cope with the demand of hundreds of thousands or even millions of test user. And it needs the support and cooperate of network operators and network distribution service provider, thus make it greatly restricted. At present, applications what we can get is often a few small scale cloud computing applications, such as private cloud testing for small enterprise.

\section{B. Hybrid Testing Strategy}

Test results of this method using the traditional performance testing tools such as LoadRunner to generate background pressure in intranet, combined with the clouds user experience, is more useful for reference than the results by independent intranet testing. But this method can't simulate a large number of the clouds pressure, cannot traverse user access paths to the clouds application to obtain the comprehensive user experience data.

\section{Intranet Testing Without The Clouds}

System test without the clouds user experience testing in the intranet, the result data can only represent the performance of the system itself and is unable to get user experience.

\section{Using Slices Testing To The Clouds Server}

This method of the inference to calculate the clouds service capacity is easy to implement, but the testing results is most difficult to be referenced.

\section{Discuss The Testing Methods}

In a word, based on the development of cloud computing service mode, the current industry within the traditional testing method has relative fell behind. The requirement for performance testing of cloud computing service is higher, such as higher pressure simulation, richer user experience data, etc. To meet this requirement, the industry also explores new testing methods.

\section{A. Super Calculate Center}

Using supercomputing center generates pressure, for performance testing of $\mathrm{CDN}$ and the system itself. Simulation load from different regions can simulate the concurrent online situation in different network environment of north and south China. High performance computer can simulate large-scale 
virtual users. But pressure, through different and complex operators' links with topological unknown, may lead to simulation load failure and bandwidth lease cost higher. For example, 1 GBPS operators bandwidth lease cost is up to hundreds of thousands Yuan.

\section{B. Producing Test Pressure Through The Clouds Is Used For Testing The Cloud Computing Applications}

This will be the direction and the idea for future cloud performance testing development. Using the existing mature network technology and virtualization technology, integrating the distributed press together in every corner of the world for performance test is also a kind of ways worth discussing. The concept of performance testing cloud platform is t testing the clouds service concept through the clouds pressure. It is the outspread of super center mode. The super calculate center is not the super computer concentration, but a lot of cheap server concentration. The clouds pressure producing platform is integrated by a large number of cheap server computing powers, such as Gomez cloud test platform [3] of Compuware company and Neolaod cloud test platform [4] of NEOTYS company, and this kind of test service price is very expensive currently.

\section{New Clouds Test Platform Is The Integration Of Open Source Software Through Secondary Development}

We can see using the clouds performance testing tools to test the clouds service performance will be a new business growth point in the future. To deal with this business growth, in addition to buy expensive commercial cloud testing tool, integrating the existing open source software test tools for secondary development is a better method, because from function of the current tools and the development of technology, we have the technical feasibility, and the cost of the clouds test platform based on open source platform will be very cheap.

\section{REFERENCES}

[1] Nomura Research Institute survey of Technology, 2012-2014 Goble Information Technology Map [J]. p:60-72.

[2] Content Delivery Network[OL]. http://baike.baidu.com/view/21895.htm

[3] Gomez[OL] . http://www.compuware.com/application-performancemanagement

[4] Neoload testing[OL]. http://www.neotys.com/ 